\title{
Musicoterapia en Medicina: Una alternativa en la rehabilitación del paciente neuroquirúrgico
}

\author{
Rafael Andrés Pájaro-Mojica',2, Rosalinda Quiroz-Mendoza ${ }^{1,2}$, Yancarlos Ramos², Alfonso Pacheco-Hernández ${ }^{2,3}$, \\ Luis Rafael Moscote-Salazar ${ }^{2,3}$ \\ 1 Estudiante de Medicina. Universidad de Cartagena. Cartagena de Indias, Colombia. \\ 2 Centro de Investigaciones Biomédicas (CIB), línea Cartagena Neurotrauma Research Group, Universidad de Cartagena, \\ Cartagena, Colombia. \\ 3 Médico. Especialista en Neurocirugía. Facultad de Medicina, Universidad de Cartagena. Cartagena de Indias, Bolívar, \\ Colombia.
}

Rev. Chil. Neurocirugía 45: 61-66, 2019

\begin{abstract}
Resumen
La música tiene un efecto positivo sobre los seres humanos. En el curso de la historia la música ha estado ligada al desarrollo de la humanidad. Diversas investigaciones han explorado el papel de la musicoterapia en lesiones cerebrales y se ha establecido su interesante efecto en diversas patologías neurológicas. Desde la mejoría en el control motor hasta la estimulación de la memoria a corto plazo, la musicoterapia se visualiza como una herramienta útil en neurocirugía para la rehabilitación de nuestros pacientes.
\end{abstract}

Palabras claves: Musicoterapia, neurorrehabilitación, neurocirugía, lesión neurológica.

\begin{abstract}
Music has a positive effect on human beings. In the course of history, music has been linked to the development of humanity. Several investigations have explored the role of music therapy in brain injuries and the interesting effect of music on various neurological pathologies has been established. From the improvement in motor control to the stimulation of short-term memory, music therapy is seen as a useful tool in neurosurgery for the rehabilitation of our patients.
\end{abstract}

Key words: Music therapy, neurorehabilitation, neurosurgery, neurological injury.

\section{Introducción}

La música ha pasado de constituir una parte importante de la cultura a convertirse en una opción terapéutica cada día más aceptada ${ }^{1,2}$. La musicoterapia, definida como el uso de la música por un profesional entrenado para lograr distintos objetivos terapéuticos, ha sido estudiada desde mucho tiempo atrás por sus efectos relajantes y curativos en los pacientes, y en la actualidad constituye un campo de la investigación neurológica y neuroquirúrgica mo- derna ${ }^{3,4}$. Los estudios actuales han permitido determinar aspectos positivos derivados del uso de la música como una terapia integral de manejo y rehabilitación de los pacientes, siendo de utilidad en el control y recuperación de los procesos cognitivos, motores, sociales, del lenguaje y del estado emocional en los pacientes con lesiones del sistema nervioso central o posterior a un procedimiento neuroquirúrgico ${ }^{4}$. Estos efectos se han asociado a la reactivación de grupos neuronales y a la regulación de zonas dentro del sistema nervioso central asociadas al proceso patológico de los pacientes ${ }^{5}$. En la actualidad se continua estudiando los beneficios derivados del uso de la musicoterapia en busca de establecerlo como una terapia definitiva dentro del campo de la medicina neuroquirúrgica moderna ${ }^{4}$.

Beneficios del estímulo musical en la funcionalidad nerviosa

Se ha demostrado la utilidad de la musicoterapia para la rehabilitación 
de pacientes con diferentes patologías como Alzheimer, Parkinson, alteraciones del estado de ánimo, o de aquellas que requieran intervenciones quirúrgicas como el trauma craneoencefálico (TCE), entre otras ${ }^{6}$. Algunos estudios en roedores han encontrado que luego de someterlos a estímulos musicales, los niveles del factor neurotrófico derivado del cerebro (BDNF) aumentan, de forma significativa, en órganos del sistema límbico (hipotálamo e hipocampo), en contraste con aquellos roedores que no son expuestos a estos estímulos ${ }^{7,8}$. Este factor está relacionado con aspectos como el crecimiento, mantenimiento y supervivencia neuronal en el sistema nervioso central (SNC); al estar disminuido en enfermedades como el Parkinson y el Alzheimer, puede indicar que la mayor capacidad de aprendizaje evidenciada en los roedores intervenidos podría ser consecuencia del incremento en la expresión de factores de crecimiento neuronales, ejerciendo así un efecto neuroprotector en el SNC ${ }^{6}$.

En estudios de neuroimagen funcional realizados en individuos sanos se ha demostrado que la música induce la activación generalizada de varias redes cerebrales y, por ende, aumenta la circulación de sangre hacia el cerebro por medio de la arteria cerebral media, creando un ambiente favorable para la recuperación del paciente ${ }^{9}$. Debido a que la rehabilitación activa basada en la música implica muchos aspectos semejantes al entrenamiento y aprendizaje musical, es de esperar que la rehabilitación neurológica con música desencadene cambios estructurales y funcionales en la neuroplasticidad similares a los presentes en sujetos sanos que han tenido alguna clase de entrenamiento musical ${ }^{10,11}$. A pesar de esto, los mecanismos celulares específicos involucrados en la neuroplasticidad inducida por la música aún se desconocen $^{9}$. Se ha demostrado que las actividades musicales facilitan la recuperación a nivel conductual y neurobiológico en modelos animales de muchas enfermedades neurológicas ${ }^{12}$.

Por otro lado, la música también juega un papel importante en la activación del sistema dopaminérgico mesolímbico encargado de la regulación de procesos como la memoria, la atención, las funciones ejecutivas, el estado de ánimo y la motivación ${ }^{13}$. En sujetos sanos, la activación del núcleo accumbens, el cual es parte importante en este sistema de recompensa, por parte de una intensa respuesta emocional a la música, conlleva a un aumento directamente proporcional en la secreción de dopamina $^{13}$. Este aumento puede explicar las ganancias cognitivo-emocionales desencadenadas por la música en pacientes con trastornos neurológicos ${ }^{9}$.

También desencadena respuestas cardiovasculares y endocrinas, las cuales pueden medirse por la baja concentración sérica de cortisol y la inhibición de las reacciones de estrés cardiovascular $^{9}$. En pacientes con ACV agudo, los niveles aumentados de cortisol se han relacionado con aumento de infartos, riesgo de depresión y pronóstico y desenlace desfavorable ${ }^{14}$, por lo cual se considera que la música puede tener un efecto beneficioso en estos pacientes al reducir los niveles de corti$\mathrm{sol}^{9}$. Además, en enfermedades en las cuales hay una disfunción del sistema motor, causando así la imposibilidad de ejecutar una secuencia o de controlar acciones, la música, por medio del arrastre rítmico, puede actuar como un temporizador, para facilitar al paciente la ejecución de movimientos y evitar la disfunción ${ }^{15}$. En pacientes con alteraciones en la coordinación posterior a un accidente cerebrovascular, pueden hallarse mejores resultados al ejecutar tareas motoras si se recurre al apoyo rítmico proporcionado por la música ${ }^{9}$.

En el campo de la neurocirugía, diversos estudios han demostrado los efectos positivos de la musicoterapia durante la realización de procedimientos quirúrgicos como, por ejemplo, craneotomías con el paciente despierto, los cuales no demostraron signos de estrés postraumático ${ }^{16}$. Para la selección del tipo de música que debe reproducirse durante este procedimiento, la mitad de los pacientes preferiría escuchar su música favorita ${ }^{16}$. Es importante que los pacientes perciban la música reproducida como "agradable" o "bella", pues aquellos quienes la perciben como "aburrida" o "desagradable" obtienen menos beneficios de ella ${ }^{17}$.

\section{Musicoterapia en pacientes con ac- cidente cerebrovascular (ACV)}

Luego de un ACV, el enfoque del cuidado durante la fase aguda es prevenir la aparición de una lesión cerebral secundaria $^{18}$. Se busca mejorar y mantener una buena perfusión cerebral, de forma prioritaria, por lo cual estos pacientes se benefician del uso de ventilación mecánica para proteger la vía aérea, garantizando una adecuada ventilación pulmonar y oxigenación sanguínea ${ }^{19}$. Los pacientes con ACV agudo que requieren ventilación mecánica, usualmente, también deben ser tratados con fármacos sedantes o hipnóticos, para facilitar sus cuidados médicos, además del monitoreo frecuente al que son sometidos para garantizar que cualquier deterioro neurológico que presenten no se deba a la medicación suministrada ${ }^{20,21}$. Esto obliga al personal asistencial a equilibrar el uso de fármacos que alteren los resultados del examen neurológico, teniendo en cuenta que la sedación causa disminución del estado de la conciencia ${ }^{19}$. El uso de terapias no farmacológicas para disminuir la ansiedad de estos pacientes, puede también reducir la duración de la estancia hospitalaria y del estrés postraumático, disminuir los costos de atención y promover una recuperación a largo plazo; es por eso que existe la posibilidad de utilizar la música como terapia en la rehabilitación de pacientes con $\mathrm{ACV}$, pues ha demostrado ser eficaz en reducir la ansiedad y el estrés de pacientes en $\mathrm{UCl}$ con ventilación mecánica ${ }^{16}$, y podría contribuir a la disminución del uso de sedantes, además de estimular la recuperación de la memoria verbal y la atención al escucharse, diariamente, durante la fase temprana post $A C V^{16,19}$. En pacientes con ACV agudo del hemisferio izquierdo, especialmente con aquellos que comprometen la arteria cerebral media, la musicoterapia diaria aumenta el volumen de la materia gris y soporta la conexión del giro frontal superior, el giro cingulado anterior y el estrato ventral ${ }^{22}$. En un estudio de casos y controles, realizado en pacientes con ACV, durante los primeros 6 meses después del evento, se evidenció que aquellos que fueron expuestos a escuchar su música favorita diariamente ( $\mathrm{n}$ =16), comparados con los pacientes en los grupos de control ( $n=18$ pacientes tratados con audiolibros, $n=15$ sin ningún tipo de material de audio), tuvieron un amplio aumento del volumen de materia gris en las conexiones de las áreas frontolímbicas, principalmente del hemisferio contralesional ${ }^{23}$. A pesar de ser beneficioso para la recuperación de pacientes críticos, el uso de la música como una intervención no ha sido 
muy bien estudiado en pacientes con ACV $^{19}$. Sin embargo, distintos estudios brindan información sobre cómo se puede beneficiar a los pacientes con ACV agudo, en estado crítico, usando la música ${ }^{19}$.

El uso de la música durante la anestesia para procedimientos quirúrgicos, brinda beneficios para la recuperación y la reducción del estrés ${ }^{24,25}$. Pacientes que durante su postoperatorio fueron expuestos a musicoterapia sumada con otras medidas terapéuticas necesitaron menos analgesia de rescate, experimentaron mayor eficacia de la misma y al momento del alta estaban menos fatigados ${ }^{24}$. En un ensayo clínico controlado aleatorizado, en el cual se pretendía evaluar el impacto de la musicoterapia intra y postoperatoria en el nivel de estrés de los pacientes y su respuesta inmune al procedimiento, se evidenció un descenso significativo en los niveles de cortisol presentes en la orina de los pacientes que fueron tratados con musicoterapia después de dos horas en la unidad de cuidados postanestésicos ${ }^{25}$. Además, el grupo expuesto a musicoterapia requirió menos analgesia que el grupo control, sin embargo, otros marcadores como la presión arterial, la glicemia, frecuencia cardíaca, saturación de oxígeno y los niveles de inmunoglobulina $A$, no presentaron ninguna variación significativa entre los grupos ${ }^{25}$.

A pesar de que no existe mucha evidencia sobre el uso de musicoterapia para el tratamiento de pacientes con ACV, esta sigue siendo utilizada por distintos miembros del equipo médico a cargo de estos pacientes y por sus familiares, quienes buscan optimizar el cuidado de los mismos ${ }^{19}$.

Beneficios de la musicoterapia en el manejo de la lesión cerebral traumática

Las lesiones cerebrales traumáticas (LCT) se caracterizan por producir en los pacientes déficit cognitivo, acompañado de alteraciones en las funciones motoras, sensitivas, conductuales y emocionales ${ }^{4}$. El estudio de la música como una opción terapéutica en los pacientes con una LCT ha sido estudiado y utilizado desde el siglo XX, siendo además un importante campo de investigación actual ${ }^{2}$. El estudio de la neuromusicología y la neurología han desarrollado nuevos métodos de tratamiento para mejorar el desarrollo cognitivo de las personas afectadas, por medio de la música como agente de neuroplasticidad y de reactivación neuronal posterior a un $\mathrm{TCE}^{4}$.

El conocimiento de los efectos beneficiosos de la musicoterapia en la atención médica ha permitido el desarrollo de nuevos procesos de rehabilitación y recuperación integral de los pacientes afectados. Algunos estudios han demostrado los efectos positivos de la musicoterapia en pacientes con lesiones cerebrales traumáticas moderadas o severas según la escala de coma de Glasgow (GCS). La exposición de los pacientes a componentes musicales de distintas características trajo como resultado la modulación de factores de alta importancia en la recuperación de los pacientes como la atención, importante en los pacientes que desarrollan pensamientos negativos en su proceso de recuperación. De igual forma, por medio de estudios imagenológicos se ha observado la actividad del sistema límbico ante el estímulo musical, regulando el desarrollo de emociones en el $\mathrm{SNC}^{2}$.

Algunas investigaciones han encontrado relaciones entre la actividad cerebral y la activación de emociones positivas o negativas durante la exposición a la música, siendo mucho más activas las áreas parahipocámpicas reguladores de las emociones positivas que las zonas de la corteza frontal ${ }^{26}$. Por otra parte, algunos aspectos fisiológicos como la alteración de la frecuencia cardiaca y de la frecuencia respiratoria ante el estímulo musical permitieron definir estilos o tipos musicales de preferencia en el paciente, regulando además el estado emocional de las personas con una lesión cerebral postraumática ${ }^{2}$. Estudios actuales han demostrado incluso una disminución considerable de la agitación en pacientes posterior a una LCT ante la exposición a su música favorita, relacionando estos resultados con la capacidad de recordar pensamientos positivos y sensaciones de placer personal al momento de escuchar la música y demostradas además por medio de la activación de zonas corticales y núcleos relacionados con la recompensa y el placer, como son el núcleo estriado ventral y la disminución de la actividad de la amígdala, relacionada con las vías neuronales del miedo y emociones negativas ${ }^{27-29}$. Además, los estudios permitieron determinar que al retirarse el estímulo de la música los pacientes tienden a aumentar de nuevo sus niveles de agitación y estrés, lo cual constituye una limitación de la musicoterapia. Como beneficio adicional, la musicoterapia permitió también la disminución de movimientos agresivos o inapropiados causados por la agitación posterior a una $\mathrm{LCT}^{28}$. La flexibilidad del proceso terapéutico derivado del uso de la música depende de muchos aspectos, entre los cuales se encuentra el tono y el ritmo musical ${ }^{30}$. La determinación de estas variables permite identificar los cambios en los procesos de atención del paciente, teniendo en cuenta que alguno de los efectos beneficiosos de la musicoterapia se ha asociado al desarrollo de complacencia del paciente. Por otra parte, estas características determinan los distintos procesos neurofisiológicos, de manera que, la participación de circuitos neuronales depende de las características mismas de la música y de su interacción con la persona expuesta 4 . Por esta razón, los estudios realizados con la exposición de los pacientes posterior a una LCT con su música favorita no dieron resultados satisfactorio en todas las personas expuestas, teniendo en cuenta que en algunos hubo un aumento de la agitación, el cual se ha relacionado con el tipo de estímulo utilizado, en el caso de tipos de música como el Rap o el metal, de ritmos fuertes y estructuras complejas $^{28}$.

La música ha mostrado además efectos potencialmente útiles desde el punto de vista terapéutico como la mejora de los procesos cognitivos, el estímulo de las actividades motoras voluntarias, con efectos beneficiosos en la marcha de los pacientes, incluyendo la fuerza y la simetría de los pasos en la rehabilitación posterior a una LCT y de los procesos comunicativos, incluyendo el habla y la capacidad de respuesta de los pacientes en la fase aguda de la lesión ${ }^{2,4}$. Se han encontrado además resultados positivos en la recuperación de la conducta adaptativa en pacientes posterior a un estado comatoso. Aunque el uso de la musicoterapia en el contexto de los pacientes comatosos ha sido poco evaluado, estudios actuales han mostrado cambios importantes en la capacidad interactiva de los pacientes y en la actividad psicomotora, aunque debe continuar siendo estudiada, para establecerla como un método de trata- 
miento activo en la rehabilitación de los pacientes comatosos ${ }^{31}$. Estos efectos se suman a los beneficios ofrecidos por la musicoterapia relacionados con la disminución de la ansiedad postquirúrgica y el estrés, incluso en el ámbito postraumático ${ }^{2}$.

La búsqueda de los procesos filológicos reguladores de la acción de la música sobre el sistema neuroquímico ha permitido identificar factores involucrados en la regulación del estrés por el eje hipotálamo-hipofisiario-adrenal ${ }^{32,33}$. Algunos estudios han relacionado la expresión de neurotransmisores como la dopamina con los efectos de la musicoterapia asociados a la sensación de placer y la motivación, además de la identificación de la serotonina y derivados de la propiomelanocortina en búsqueda de una posible mediación inmunológica derivada de la exposición a la música ${ }^{4,34,35}$. Los efectos placenteros identificados en la utilización de la música como agente terapéutico han mostrado activación de zonas corticales, mesolímbicas y del núcleo accumbens, implicadas en el manejo de las emociones ${ }^{36,37}$. Se ha buscado además, destacar la influencia de la música sobre factores sociales y de resocialización, y la relación neuroquímica de estos aspectos con la expresión de factores neurobiológicos como la oxitocina ${ }^{4,34}$. Por otra parte, los estudios actuales han planteado nuevas teorías relacionadas con la capacidad de la música de estimular procesos de neurotrofismo y neuroplasticidad, aunque no se han investigado factores como el factor neurotrófico derivado del cerebro $(\text { FNDC })^{4,5}$. Sin embargo, a pesar de que el conocimiento sobre la neuroplasticidad inducida por la musicoterapia es limitado, se han desarrollado estudios basados en la musicoterapia y la producción de música en el contexto de pacientes con LCT. Dykesteen et al., ha investigado la producción de música y su relación con la recuperación neurológica, teniendo resultados positivos consistentes en aumento de la actividad neuronal y de la funcionalidad cognitiva de los pacientes. En algunos pacientes incluidos en el estudio se observaron cambios positivos en la corteza orbitofrontal, la cual regula distintos procesos cognitivos relacionados con el comportamiento, la atención y la capacidad de tomar decisiones ${ }^{38}$.

\section{Estudios actuales de la musicotera- pia en el ámbito neuroquirúrgico}

La musicoterapia ha permitido establecer nuevos métodos para el tratamiento de distintas patologías neuroquirúrgicas, y ha abierto nuevas posibilidades en la rehabilitación de procedimientos quirúrgicos de la medula espinal. Estudios actuales han permitido observar los efectos beneficiosos de la musicoterapia en el periodo postoperatorio de procedimientos en la columna vertebral, siendo un punto a destacar la disminución del dolor postoperatorio ${ }^{39}$. Estos aspectos son relevantes clínicamente teniendo en cuenta que plantean una opción terapéutica no farmacológica, lo cual podría determinar una opción de tratamiento con pocos o ningún efecto adverso y a su vez contribuye a la actividad temprana del paciente, regulando los estados prolongados de sedación u otros efectos adversos que dificultan la actividad del personal de atención ${ }^{39,40}$

\section{Musicoterapia y tumores del sistema nervioso}

En la actualidad no hay suficiente evidencia respecto al uso de la musicoterapia en pacientes con tumores del sistema nervioso durante el periodo perioperatorio, a pesar de que se han mostrado efectos positivos en el manejo de otros tipos de cáncer ${ }^{41}$. El potencial beneficio teórico derivado del uso de la musicoterapia se basa en la capacidad de disminuir la ansiedad y el estrés en medio de los procedimientos quirúrgicos del sistema nervioso central, características que han sido probadas en distintos estudios clínicos. Un estudio realizado en pacientes que iban a recibir cirugía cerebral, investigó los efectos de musicoterapia en este grupo utilizando una escala análoga visual. Cada paciente recibió una tera- pia de 20 a 30 minutos de música de su preferencia en vivo, brindada por un musicoterapeuta antes del acto quirúrgico y seguida por una sesión diaria durante su recuperación. Este estudio encontró una diferencia significativa en los niveles de ansiedad, la percepción de la estancia hospitalaria, relajación y estrés, en aquellos que dieron mayor calificación a la terapia ${ }^{42}$. Sin embargo, debe ser estudiado con mayor amplitud los probables usos de la musicoterapia en el manejo de tumores del sistema nervioso, en búsqueda de nuevas posibilidades terapéuticas.

\section{Conclusiones}

La evidencia actual indica la utilidad de la musicoterapia en la rehabilitación y recuperación de los pacientes posterior a lesiones del sistema nerviosos central de distintas características. De igual forma, se ha demostrado la efectividad de la música como agente neuroprotector en el SNC, estimulando la neuroplasticidad y regulando los procesos nerviosos que intervienen en la memoria, los procesos cognitivos, la atención, las funciones ejecutivas, el estado de ánimo y la motivación. La musicoterapia también favorece a aquellos pacientes con enfermedades neurológicas que ocasionen alteración del sistema motor, pues por medio del arrastre rítmico, puede facilitarle al paciente la ejecución de movimientos secuenciales. Además de lo anterior, se ha evidenciado los potenciales efectos de la musicoterapia en la disminución del estrés y la ansiedad posterior a una lesión o una intervención neuroquirúrgica, contribuyendo además a la disminución del uso de sedantes e hipnóticos en los procedimientos quirúrgicos. Se debe continuar estudiando los efectos de la musicoterapia en patologías neuroquirúrgicas, además de las posibles intervenciones del estímulo musical en el manejo de los tumores de sistema nervioso central.

Recibido: 20 de agosto de 2018 Aceptado: 24 de octubre de 2018 


\section{Referencias}

1. Manuel J, Rojas O. Efecto ansiolítico de la musicoterapia: aspectos neurobiológicos y cognoscitivos del procesamiento musical. Rev Colomb Psiquiat. 2011; 40(4): 748-59.

2. Reddy B, Phanisree P, Priyanka M, Kavitha D, Indira S, Bhandarkar P, et al. Effect of Music Therapy in Patients with Moderate - to - severe Traumatic Brain Injury. J Datta Meghe Inst Med Sci Univ. 2017; 12: 51-4.

3. Martin-Saavedra J, Vergara-Méndez L, Talero-Gutiérrez- C. Music is an effective intervention for the management of pain: An umbrella review. Complement Ther Clin Pract. 2018; 32: 103-14.

4. Hegde S. Music-based cognitive remediation therapy for patients with traumatic brain injury. Front Neurol. 2014; 5(34): 1-7.

5. Fukui H, Toyoshima K. Music facilitate the neurogenesis, regeneration and repair of neuron. Med Hypotheses. 2008; 71: 765-9.

6. Rojas JMO. Efecto ansiolítico de la musicoterapia: aspectos neurobiológicos y cognoscitivos del procesamiento musical. Rev Colomb Psiquiatr. 2011; 40(4): 748-59.

7. Angelucci F, Ricci E, Padua L, Sabino A, Tonali PA. Music exposure differentially alters the levels of brain-derived neurotrophic factor and nerve growth factor in the mouse hypothalamus. Neurosci Lett. 2007; 429(2-3): 152-5.

8. Angelucci F, Fiore M, Ricci E, Padua L, Sabino A, Tonali PA. Investigating the neurobiology of music: Brain-derived neurotrophic factor modulation in the hippocampus of young adult mice. Behav Pharmacol. 2007; 18(5-6): 491-6.

9. Sihvonen AJ, Särkämö T, Leo V, Tervaniemi M, Altenmüller E, Soinila S. Music-based interventions in neurological rehabilitation. Lancet Neurol. 2017; 16(8): 648-60.

10. Schlaug G. Musicians and music making as a model for the study of brain plasticity. 1st ed. Vol. 217, Progress in Brain Research. Elsevier B.V.; 2015. 37-55 p.

11. Wan CY, Schlaug G. Music making as a tool for promoting brain plasticity across the life span. Neuroscientist. 2010; 16(5): 566-77.

12. Baroncelli L, Braschi C, Spolidoro M, Begenisic T, Sale A, Maffei L. Nurturing brain plasticity: Impact of environmental enrichment. Cell Death Differ. 2010; 17(7): 1092-103.

13. Salimpoor VN, Benovoy M, Larcher K, Dagher A, Zatorre RJ. Anatomically distinct dopamine release during anticipation and experience of peak emotion to music. Nat Neurosci. 2011; 14(2): 257-64.

14. Barugh AJ, Gray P, Shenkin SD, MacLullich AMJ, Mead GE. Cortisol levels and the severity and outcomes of acute stroke: A systematic review. J Neurol. 2014; 261(3):533-45.

15. Nombela C, Hughes LE, Owen AM, Grahn JA. Into the groove: Can rhythm influence Parkinson's disease? Neurosci Biobehav Rev. 2013; 37(10): 2564-70.

16. Gasenzer ER, Kanat A, Neugebauer E. Neurosurgery and Music; Effect of Wolfgang Amadeus Mozart. World Neurosurg. 2017;102:3139.

17. Jadavji-Mithani R, Venkatraghavan L, Bernstein M. Music is beneficial for awake craniotomy patients: A qualitative study. Can J Neurol Sci. 2015; 42(1): 7-16.

18. Olson DWM, Graffagnino C. Consciousness, coma, and caring for the brain-injured patient. AACN Clin Issues. 2005; 16(4): 441-55.

19. Supnet C, Crow A, Stutzman S, Olson DW. Music as medicine: The Therapeutic Potential of Music for Acute Stroke Patients. Crit Care Nurse. 2016; 36(2): e1-7.

20. Olson DWM, Graffagnino C, King K, Lynch JR. Toward solving the sedation-assessment conundrum: Bispectral index monitoring and sedation interruption. Crit Care Nurs Clin North Am. 2005; 17(3): 257-67.

21. Jacobi J, Fraser GL, Coursin DB, Riker RR, Fontaine D, Wittbrodt ET, et al. Analgesics in the Critically III Adult. Crit Care Med. 2002; 30(1): 119-41.

22. Särkämö T, Altenmüller E, Rodríguez-fornells A. Editorial: Music , Brain , and Rehabilitation: Emerging Therapeutic Applications and Potential Neural Mechanisms. 2016; 10(March): 1-5.

23. Särkämö T, Ripollés P, Vepsäläinen H, Autti T, Silvennoinen HM, Salli E, Laitinen S, Forsblom A, Soinila S, Rodríguez-Fornells A. Structural Changes Induced by Daily Music Listening in the Recovering Brain after Middle Cerebral Artery Stroke: A Voxel-Based Morphometry Study. Front Hum Neurosci. 2014; 8(April): 1-16.

24. Nilsson U, Rawal N, Uneståhl LE, Zetterberg C, Unosson M. Improved recovery after music and therapeutic suggestions during general anaesthesia: a double-blind randomised controlled trial. Analgesia following music and therapeutic suggestions in the PACU in ambulatory surgery; a randomized controlled trial. 2001; 45(7): 1-7.

25. Nilsson U, Unosson M, Rawal N. Original Article Stress reduction and analgesia in patients exposed to calming music postoperatively: a randomized controlled trial. 2005; 96-102.

26. Kleinstauber M, Gurr B. Music in brain injury rehabilitation. J Cogn Rehabil. 2006; 24: 4-14.

27. Sarkamo T, Tervaniemi M, Laitinen S, Forsblom A, Soinila S, Mikkonen M, et al. Music listening enhances cognitive recovery and mood after middle cerebral artery stroke. Brain. 2008; 131: 866-76.

28. Park S, Williams R, Lee D. Effect of Preferred Music on Agitation After Traumatic Brain Injury. West J Nurs Res. 2015; 38(4): 1-17.

29. Van de Winckel A, Feys H, De Weerdt W, Dom R. Cognitive and behavioral effects of music-based exercises in patients with dementia. Clin Rehabil. 2004; 18: 253-60.

30. Peretz I. The nature of music from a biological perspective. Cognition. 2006; 100: 1-32.

31. Formisano R, Vinicola V, Penta F, Matteis M, Brunelli S, Weckel JW, et al. Active music therapy in the rehabilitation of severe brain injured patients during coma recovery. Ann Ist Super Sanità. 2001; 37(4): 627-30.

32. Koelsch S, Fuermetz J, Sack U, Bauer K, Hohenadel M, Wiegel ME al. Effects of music listening on cortisol levels and propofol consumption during spinal anaesthesia. Front Psychol. 2011; 2(58): 1-9.

33. Suda M, Morimoto K, Obata A, Koizumi H, Maki A. Emotional responses to music: towards scientific perspectives on music therapy. Neuroreport. 2008; 19(1): 75-8.

34. Chanda M, Levitin D. The neurochemistry of music. Trends Cogn Sci. 2013; 17: 179-93.

35. Salimpoor V, Benovoy M, Larcher K, Dagher A, Zatorre R. Anatomically distinct dopamine release during anticipation and experience of peake motion to music. Nat Neurosci. 2011; 14: 257-62.

36. Menon V, Levitin D. The rewards of music listening: response and physiological connectivity of the mesolimbic system. Neuroimage. 2005; 28: 175-84.

37. Salimpoor V, Van den Bosch I, Kovacevic N, McIntosh A, Dagher A, Zatorre R. Interactions between the nucleus accumbens and auditory cortices predict music reward value. Science (80- ). 2013; 340: 216-9.

38. Marie B, Vik D, Skeie GO, Vikane E, Specht K. Effects of music production on cortical plasticity within cognitive rehabilitation of patients 
with mild traumatic brain injury. Brain Inj. 2018; 32(5): 634-43.

39. Homel P, Lonner B, Shepp J, Lichtensztejn M, Loewy J V. Music Therapy Increases Comfort and Reduces Pain in Patients Recovering From Spine Surgery. Am J Orthop. 2017; 46(1): 13-22.

40. Kleiber C, Adamek M. Adolescents ' perceptions of music therapy following spinal fusion surgery. JCN. 2012; 22: 1-9.

41. Rossetti A, Chadha M, Torres BN, Lee JK, Hylton D, Loewy J V., et al. The Impact of Music Therapy on Anxiety in Cancer Patients Undergoing Simulation for Radiation Therapy. Int J Radiat Oncol. 2017; 99(1): 103-10.

42. Walworth D, Rumana CS, Nguyen J, Jarred J. Effects of live music therapy sessions on quality of life indicators, medications administered and hospital length of stay for patients undergoing elective surgical procedures for brain. J Music Ther. 2008; 45(3): 349-59.

\section{Correspondencia a:}

Dr. Luis Rafael Moscote-Salazar

mineurocirujano@aolcom 